\title{
JOINT DISCUSSION
}

\section{REFERENCES}

[I] Fields, Studier, Diamond, Mech, Inghram, Pyle, Stevens, Freid, Manning, Ghiorso, Thompson, Higgins and Seaborg. Phys. Rev. ro2, 180, 1956.

[2] Burbidge, E. M., Burbidge, G. K., Fowler, W. A. and Hoyle, F. Rev. Mod. Phys. 29, 547, 1957.

\section{NEUTRON PROCESSES IN GIANT STARS}

\section{A. G. W. CAMERON}

A red giant star is believed to be one in which the central regions have been exhausted of hydrogen. When helium reactions commence in such a star there are two potential sources of neutrons.

I. The ${ }^{13} \mathrm{C}$ hypothesis. When helium reactions start forming ${ }^{12} \mathrm{C}$ in a degenerate helium core, the core must expand and rather a lot of energy is liberated. It is possible that the resulting short-lived extensive convection zone will mix some hydrogen from the envelope with ${ }^{12} \mathrm{C}$ produced in the core to form ${ }^{13} \mathrm{C}$ by the reactions ${ }^{12} \mathrm{C}(p, \gamma){ }^{13} \mathrm{~N}(\beta+\nu){ }^{13} \mathrm{C}$. This can later be consumed by ${ }^{13} \mathrm{C}(\alpha, n){ }^{16} \mathrm{O}$ reaction [x], producing neutrons which are slowed to thermal equilibrium and then captured by surrounding nuclei. Very little production of heavy elements occurs unless the mixing ratio of protons to ${ }^{12} \mathrm{C}$ is of the order of $0 \cdot I$ by number [2].

2. The ${ }^{21} \mathrm{Ne}$ hypothesis. In the red giant star the temperature of the hydrogen shell source is probably high enough to produce a considerable amount of ${ }^{21} \mathrm{Ne}$ by the reactions ${ }^{20} \mathrm{Ne}(p, \gamma){ }^{21} \mathrm{Na}\left(\beta^{+} \nu\right){ }^{21} \mathrm{Ne}[3]$. It is not known how much ${ }^{21} \mathrm{Ne}$ will be destroyed by the ${ }^{21} \mathrm{Ne}(p, \gamma){ }^{22} \mathrm{Na}(\beta+\nu){ }^{22} \mathrm{Ne}$ reactions. The surviving ${ }^{21} \mathrm{Ne}$ later produces neutrons by the ${ }^{21} \mathrm{Ne}(\alpha, n){ }^{24} \mathrm{Mg}$ reaction.

Whatever the source of neutrons, the resulting process may be called neutron capture on a slow time-scale (Burbidge $e t$ al. have abbreviated this to $s$-process [4]). The early neutrons are captured predominantly by the nuclei of the iron abundance peak. Continuing neutron capture (interspersed with beta-decays) then drives some of the nuclei toward the heavy element region. The heavy element abundances become about three orders of magnitude larger than those originally present in the star [2]. Heavy element synthesis terminates at lead and bismuth because further neutron capture leads to shortlived nuclei which decay by alpha-particle emission. Only a small amount of neutronevolved material need be mixed into stellar surface layers to produce the characteristic over-abundances of $\mathrm{S}$ and $\mathrm{Ba}$ II stars.

A brief summary was also given of the speaker's recent work on super-nova explosions: A very massive star is considered in an advanced stage of evolution. It is postulated that there is a degenerate core composed of the nuclei in the iron equilibrium peak at a temperature not much in excess of $2 \times \mathrm{IO}^{9}{ }^{\circ} \mathrm{K}$. This is surrounded by successive layers of intermediate elements, carbon, helium, and hydrogen. The intermediate layer also contains heavy elements made by neutron capture on a slow time-scale through a series of reactions accompanying carbon consumption (not described above).

As the mass of the central core increases, the central density becomes very large, and the Fermi level of the electrons becomes several $\mathrm{MeV}$. The iron nuclei capture some of the electrons and are driven away from the valley of beta-stability. At a density of about Io ${ }^{11} \mathrm{~g} / \mathrm{cm}^{3}$ the electron capture products become unstable to neutron emission. The core then collapses and most of it is converted to neutrons.

The outer layers also implode, but very little nuclear activity takes place in the hydrogen and helium layers. The carbon layer is mostly converted to oxygen and magnesiumregion nuclei. The remainder of the material is converted to nuclei of the silicon-calcium region. The heavy elements mostly survive intact, but some of them suffer photodisintegration, forming 'by-passed' heavy nuclei. The energy released by these transformations explodes the envelope away from the core. 


\section{NUCLEOGENESIS IN STARS}

The core is extremely hot and lies in a deep potential hole. The outer iron material is evaporated and freezes very quickly, preserving the iron abundance distribution at a high characteristic equilibrium temperature. Evaporation of the iron-neutron transition region produces neutron-rich nuclei by neutron capture on a fast time-scale. In very massive super-novae (type II?) the neutron core is too hot to contain seed nuclei; when some of it evaporates only deuterium and helium can be formed. Less massive super-novae (type I?) may contain seed nuclei in the neutron core and so produce large amounts of heavy elements by neutron capture on a fast time-scale, including the special nucleus ${ }^{254} \mathrm{Cf}$.

The type II super-nova described above can thus be expected to produce nearly the entire solar system abundance distribution of the elements in the correct relative proportions.

\section{REFERENCES}

[I] Cameron, A. G. W. Ap. J. I2I, I44, I955.

[2] Cameron, A. G. W. 'Stellar Evolution, Nuclear Astrophysics, and Nucleogenesis.' Chalk River Report, CRL-4I, 1957.

[3] Fowler, W. A., Burbidge, G. R. and Burbidge, E. M. Ap. J. 122, 271, 1955.

[4] Burbidge, E. M., Burbidge, G. R., Fowler, W. A. and Hoyle, F. Rev. Mod. Phys. 29, 547. 1957.

\section{OBSERVATIONS ON ELEMENT ABUNDANCES IN STARS}

\section{E. MARGARET BURBIDGE ANDG. R, BURBIDGE}

The basic astronomical information relating to the synthesis of the elements in stars is the determination of abundances of the elements throughout as large a sample of the universe as possible. The information available may be divided into three main parts.

I. The first consists of abundances in the Sun and solar system, supplemented by other nearby stars. These provide a so-called ' local sample'. Detailed study of them has formed the basis for the theory of the origin of the elements in stars [I] outlined by Professor Fowler in his introductory paper.

2. The second part concerns abundances in stars of different ages and locations in the Galaxy. If hydrogen alone is primeval, the oldest stars in our Galaxy today would be expected to contain the smallest proportion, relative to hydrogen, of elements heavier than hydrogen.

3. The third division of the observational material consists of the determinations of relative abundances in individual peculiar stars, in whose interiors nuclear reactions have been occurring. These provide the most direct observational evidence for the theory. Such stars will show over-abundances, relative to the normal or average, of those elements which have been built in their interiors, and an under-abundance of hydrogen if this has been consumed. For such effects to become visible on the surface, mixing between the core and envelope of the star must have occurred, and this only happens at a fairly late stage in a star's evolutionary history.

4. To these three main divisions may be added a fourth, perhaps less intimately connected with stellar evolution, but of great intrinsic interest. This concerns the anomalous abundances determined in magnetic $A$ - and F-type stars by us [2] and by Margharita Hack [3]. According to the work by Fowler and us [4] these are thought to be localized on the stellar surface and to be due to nuclear reactions initiated by magnetically accelerated particles.

To return to the first sub-division, the Sun provides evidence on only about half of the first 83 elements up to bismuth. Relative abundances of the isotopes, which are so much more informative than element abundances alone for constructing a theory, cannot be 